ORIGINAL ARTICLE

\title{
Cyclooxygenase 2 expression in nasopharyngeal carcinoma: immunohistochemical findings and potential implications
}

\section{K-B Tan, T C Putti}

J Clin Pathol 2005;58:535-538. doi: 10.1136/icp.2004.021923

See end of article for authors' affiliations

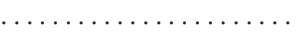

Correspondence to: Dr K-B Tan, Department of Pathology, National University of Singapore Lower Kent Ridge Road, Singapore 119074; pattankb@nus.edu.sg

Accepted for publication 25 October 2004

Background: Cyclooxygenase 2 (COX-2), an inducible prostaglandin synthase, participates in inflammatory and neoplastic processes. It is expressed by various tumours and contributes to carcinogenesis. Notably, COX-2 inhibitors appear to have tumour suppressor effects and are being evaluated in clinical trials.

Aims: To investigate COX-2 expression in nasopharyngeal carcinoma (NPC), a common tumour in parts of Asia, and to discuss potential implications.

Methods: Eighty five cases of NPC were reviewed. COX-2 immunohistochemistry and semiquantitative assessment of expression in nasopharyngeal biopsies were performed. Because COX-2 is proangiogenic, tumour microvessel density was also assessed with the use of CD31 immunohistochemistry.

Results: Histologically, 78 NPCs were undifferentiated, six were non-keratinising, and one was keratinising. Thirty nine NPCs had adjacent dysplastic epithelium. COX-2 expression was noted in 60 NPCs, 14 of 39 samples of dysplastic epithelium, and only one of 25 samples of normal epithelium $(p<0.01)$. Microvessel density was not significantly different between COX-2 positive and COX-2 negative tumours $(p=0.774)$. Tumour COX-2 positivity was not associated with higher tumour stage ( $\mathrm{p}=0.423$ ).

Conclusion: COX-2 expression is more frequently seen as nasopharyngeal epithelium progresses from normal to dysplastic to carcinoma. This suggests that COX-2 contributes to the multistep process of NPC carcinogenesis. COX-2 represents a therapeutic target for COX-2 inhibitors, and there is thus a basis for the further investigation of this adjuvant treatment modality for NPC. COX-2 inhibitors are known to potentiate the antitumour effects of radiotherapy, which is the primary treatment for NPC.

$\mathrm{C}$ clooxygenase $(\mathrm{COX})$ is a key enzyme involved in prostaglandin production. There are two forms: COX-1 and COX-2. COX-1 is expressed constitutively on cell membranes in normal tissue, whereas COX-2 is inducible during inflammatory and neoplastic processes. ${ }^{1}$ COX-2 is expressed by many tumours and there is growing evidence that it contributes to carcinogenesis. ${ }^{2}$ It has been reported to modulate xenobiotic metabolism, to promote tumour growth, invasiveness, and angiogenesis, and to inhibit apoptosis. Many experimental and clinical models have also shown that COX-2 inhibitors suppress tumour formation, ${ }^{2-4}$ and a COX-2 inhibitor (Celecoxib) has recently been approved by the Food and Drug Administration for the adjuvant treatment of familial adenomatous polyposis.

"COX-2 is expressed by many tumours and there is growing evidence that it contributes to carcinogenesis"

Nasopharyngeal carcinoma (NPC) occurs sporadically in the West, but ranks among the top 10 cancers in southern China, Taiwan, and parts of South East Asia, including Singapore. ${ }^{5}$ Many aetiological factors, both genetic and environmental, have been implicated, key among which is Epstein-Barr virus (EBV) latent infection. ${ }^{6}$ A recent study on a small number of NPCs has shown that EBV can induce COX-2, which in turn caused increased expression of vascular endothelial growth factor. ${ }^{7}$ Apart from this study, there are limited data on COX-2 expression in NPCs. We have investigated COX-2 immunoexpression in NPC and its adjacent nasopharyngeal epithelium to increase our understanding of the role of COX-2 in NPC carcinogenesis and as a potential treatment target for COX-2 inhibitors. Because COX-2 is closely linked to angiogenesis, we also studied the association of COX-2 expression with tumour microvessel density (MVD).

\section{METHODS}

\section{Patients}

We studied 85 cases of NPC diagnosed from 1997 to 2000 via nasopharyngeal biopsies at the department of pathology, National University Hospital, Singapore. The ages of the patients ranged from 22 to 80 years (mean, 49.1); 76 were ethnic Chinese, seven were Malays, and two were Indians. Our study was approved ethically by the institutional review board of the National University of Singapore. The patients' clinical records were reviewed: the tumour TNM staging ${ }^{8}$ and EBV serology were recorded, and either an EBV viral capsid antigen $\operatorname{IgA}$ of $>10$ or EBV early antigen IgA of $>5$ was classified as a raised EBV IgA titre.

\section{Immunohistochemistry}

Microwave antigen retrieval was performed in citrate buffer ( $\mathrm{pH}$ 6.0) for 20 minutes. Endogenous peroxidase was blocked with $0.6 \%$ hydrogen peroxide in methanol for 30 minutes. Incubation with anti-COX-2 (Cayman Chemical, Ann Arbor,

Abbreviations: COX, cyclooxygenase; EBV, Epstein-Barr virus; MVD, microvessel density; NPC, nasopharyngeal carcinoma 


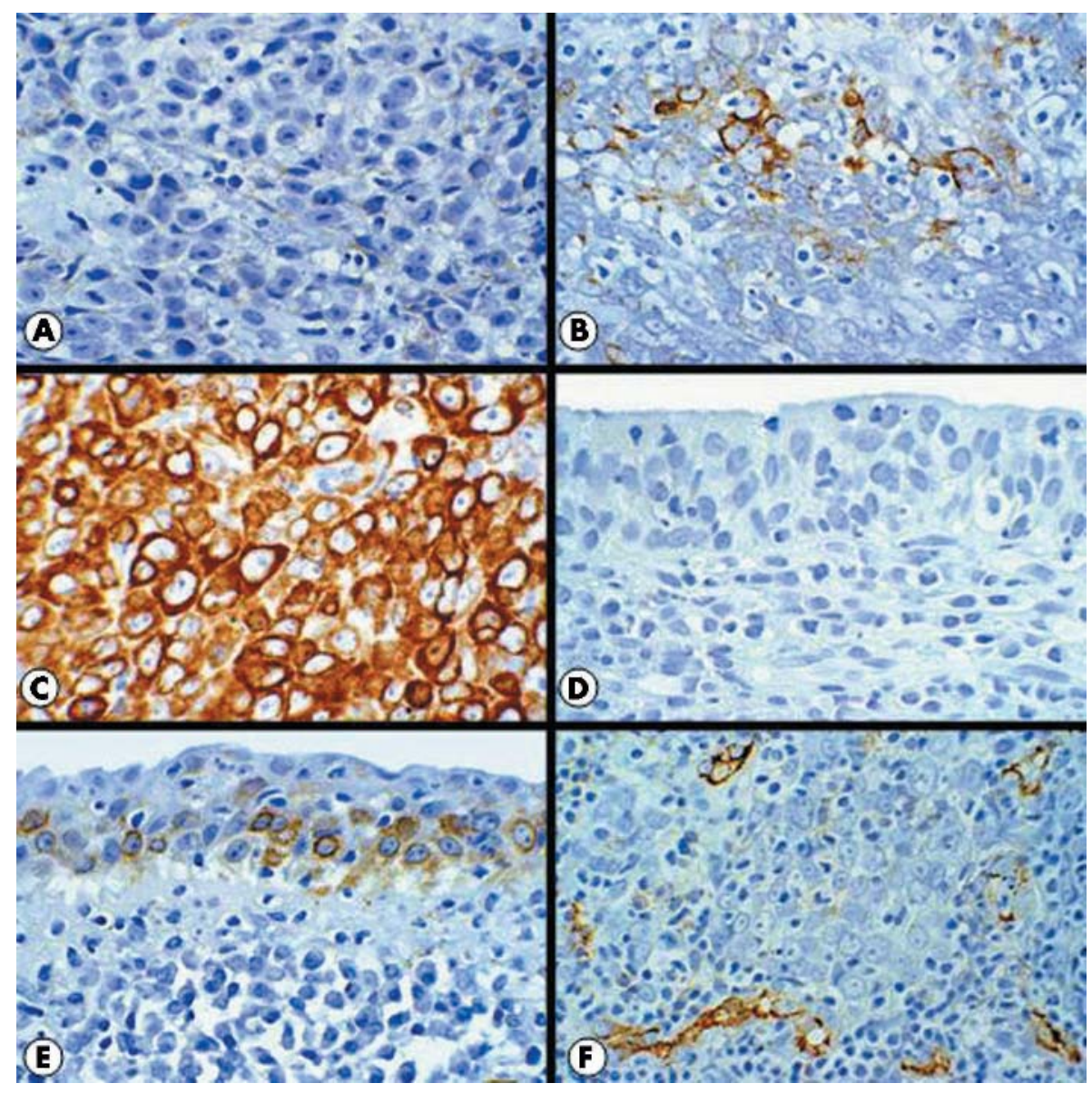

Figure 1 (A) Weak (intensity: 1 out of 3), (B) moderate (intensity: 2 out of 3), and (C) strong (intensity: 3 out of 3) COX-2 (cyclooxygenase 2) staining in separate cases of nasopharyngeal carcinoma. (D) No COX-2 staining in an area of normal nasopharyngeal epithelium. (E) Moderate COX-2 staining in an area of dysplastic nasopharyngeal epithelium. (F) CD31 highlighted microvessels, within and immediately adjacent to tumour tissue. Immunoperoxidase staining; original magnification: $(A-E), \times 600 ;(F), \times 400$.

Michigan, USA) and anti-CD31 (Dako A/S, Glostrup, Denmark) was carried out overnight at room temperature. Thereafter, further processing involved the use of the EnVision kit and protocol (Dako A/S).

\section{Assessment of immunostaining}

All fields of the immunoperoxidase slides were examined by the two authors concomitantly, using a double headed microscope. Semiquantitative assessment of COX-2 immunostaining was done by consensus and comprised both intensity of staining $(0,1,2$, or 3 ) (fig $1 \mathrm{~A}-\mathrm{C}$ ) and extent of staining $(0,0 \% ; 1,<10 \% ; 2,10-50 \% ; 3,>50 \%)$. The scores for the intensity of staining and extent of staining were multiplied to give a weighted COX-2 score for each case (maximum possible, 9). All cases with at least moderate staining intensity ( 2 or 3 ) in a minimum of $10 \%$ of the tumour cells were regarded as COX-2 positive (weighted score of $\geqslant 4$ out of 9). COX-2 immunostaining in the adjacent nasopharyngeal epithelium, both normal and dysplastic, was similarly assessed (fig ID, E). A tissue section of a case of invasive breast carcinoma was used as a positive control. For the MVD assessment using the CD31 endothelial marker, only microvessels within and in the immediate vicinity of the tumour tissue were counted. Tumour microvasculature was initially assessed at lower power $(\times 100$ magnification), and areas with the highest number of microvessels were then assessed at high power $(\times 400$ magnification) for each case (MVD) (fig IF).

\section{Statistics}

Fisher's test was used to assess differences in proportions. The $\chi^{2}$ test for independence was used to examine a possible relation between tumour subtype and COX-2 expression. The statistical software used was StatXact 6.0 (Cytel Software Corporation, Massachusetts, USA). The two tailed student's $t$ test (Microsoft Excel 2000) was used to compare the mean MVD scores between the COX-2 positive and negative tumour groups.

\section{RESULTS}

Histologically, 78 of the tumours were undifferentiated carcinomas, six were non-keratinising poorly differentiated carcinomas, and only one was a keratinising squamous cell carcinoma. After review, 39 of the 85 NPCs were also noted to have adjacent dysplastic epithelium (carcinoma in situ), as assessed by the presence of cytological and architectural atypia that affected the whole thickness of the epithelium. The cytological atypia incorporated nuclear enlargement and irregularity, with prominent nucleoli. ${ }^{9}$

COX-2 expression was seen in 60 NPCs, 14 of the 39 samples of dysplastic epithelium, and one of the 25 samples of normal epithelium. Table 1 summarises these results. For the subtypes of NPC, COX-2 positivity was noted in 54 of 78 cases of undifferentiated carcinoma and in all six cases of non-keratinising carcinoma. The only case of keratinising NPC was COX-2 negative. The differences between these proportions were just short of significance $(p=0.058)$ 
Table 1 Comparison of proportions of COX-2 positivity in NPC, dysplastic epithelium, and normal epithelium

\begin{tabular}{|c|c|c|}
\hline $\begin{array}{l}\text { Type of epithelium } \\
\text { (n) }\end{array}$ & $\begin{array}{l}\text { Number of COX-2 } \\
\text { positive cases (\%) }\end{array}$ & p Value \\
\hline NPC (85) & $60(71 \%)$ & \\
\hline $\begin{array}{l}\text { Dysplastic epithelium } \\
\text { (39) }\end{array}$ & $14(36 \%)$ & 0.0004 \\
\hline $\begin{array}{l}\text { Normal epithelium } \\
\text { (25) }\end{array}$ & $1(4 \%)$ & 0.0051 \\
\hline
\end{tabular}

The relations between COX-2 positivity in NPC and the adjacent epithelium, both dysplastic and normal, were also assessed. Sixty four cases of NPC had identifiable adjacent epithelium. Thirteen of 43 COX-2 positive NPCs also had COX-2 positive adjacent epithelium, whereas only two of 21 COX-2 negative NPCs had COX-2 positive adjacent epithelium; however, this difference was not significant $(\mathrm{p}=0.114)$.

The MVD ranged from 0 to 64 (mean, 25.2) in the COX-2 negative NPCs, which was not significantly different from the MVD range of 1 to 59 (mean, 24.2) seen in the COX-2 positive NPCs $(\mathrm{p}=0.774)$.

EBV serology results were available in 13 of 25 COX-2 negative cases and in 27 of 60 COX-2 positive cases. The incidence of EBV serological positivity was similar in the COX-2 negative (12 of 13) and COX-2 positive (25 of 27) cases.

Tumour TNM staging information was available in 79 cases. In the COX-2 negative group, nine patients were stage I or II and 13 were stage III or IV. In the COX-2 positive group, 17 patients were stage I or II and 42 were stage III or IV. There was no significant association between COX-2 expression and higher tumour stage $(\mathrm{p}=0.423)$.

\section{DISCUSSION}

Our study showed that a high proportion (60 of 85) of NPCs expressed COX-2. The proportion of cases expressing COX-2 increased as the nasopharyngeal epithelium progressed from normal to dysplastic to carcinoma. Consistent with the notion that COX-2 contributes to various carcinogenetic processes, our observations suggest that COX-2 plays a role in NPC carcinogenesis. It is possible that COX-2 is involved in the formation of dysplastic nasopharyngeal epithelium and in the progression of dysplastic epithelium to invasive lesions, at least in some cases.

The concept of dysplastic nasopharyngeal epithelium as a precursor to NPC is in keeping with precursor lesions seen in other carcinomas. In practice, however, such lesions are not easy to recognise. Several different synonyms have been used: atypia, dysplasia, carcinoma in situ, and nasopharyngeal intraepithelial neoplasia. ${ }^{9-11}$ In various NPC series the incidence of these lesions has been reported to be between $3 \%$ and $73 \% .{ }^{10}{ }^{11}$ This large variability attests to the suboptimal recognition of this entity, mainly because of the absence of widely accepted diagnostic criteria.

\section{"Consistent with the notion that COX-2 contributes to various carcinogenetic processes, these observations suggest that COX-2 plays a role in nasopharyngeal carcinoma carcinogenesis"}

The clinical implications of the presence of dysplastic nasopharyngeal epithelium are uncertain. A report by Pak et al documented the subsequent development of invasive NPC in two of three patients initially diagnosed with NPC in situ. ${ }^{12}$ Our finding of COX-2 expression in an intermediate proportion of dysplastic samples supports the neoplastic nature of this lesion and its possible invasive potential. A study of NPCs by Sheu et al also showed that an intermediate proportion of dysplastic samples expressed bcl-2. ${ }^{13}$ Interestingly, both $\mathrm{COX}-2$ and bcl-2 are antiapoptotic proteins and are closely associated in this regard. ${ }^{14}$ Further investigations into the biology and behaviour of nasopharyngeal dysplasia are required.

We noted a trend for the epithelium adjacent to COX-2 positive tumours also to be COX-2 positive. This phenomenon has also been seen in carcinomas of the breast, colon, and lung, and has been attributed to a field effect. ${ }^{15}{ }^{16}$ Some authors have suggested that such adjacent non-neoplastic epithelium may be part of the disease process, and thus deserve further study as a marker for disease recurrence and as a potential therapeutic target. ${ }^{16}$

We found no clear association between NPC histological subtype and COX-2 expression. This may be because of the small number of non-keratinising poorly differentiated NPCs and keratinising NPCs in our series. It would be interesting to study COX-2 expression in Western populations, which have a lower overall incidence of NPC, but appear to have a greater proportion of keratinising NPCs. ${ }^{17}{ }^{18}$ This subtype of NPC has been reported to have a worse prognosis, probably because of its relative radioresistance..$^{17}$

COX-2 promotes angiogenesis whereas COX-2 inhibitors have been shown to be antiangiogenetic. ${ }^{19}{ }^{20}$ Murono et al showed that EBV latent membrane protein 1 induced COX-2 expression, which in turn led to increased vascular endothelial growth factor production in NPC. ${ }^{7}$ Surprisingly, we could not find an association between COX-2 expression and MVD in our series. One important reason could have been the limited sample size of the nasopharyngeal biopsies. In several studies that were able to demonstrate such a positive association, the tissue sections examined were probably sizable because the patients had undergone tumour resection. ${ }^{21}{ }^{22}$ Another problem encountered in some cases was the presence of areas of smudged and diffuse CD31 staining. Such areas precluded the accurate counting of microvessels and had to be disregarded, which may have introduced some bias.

The high incidence of positive EBV serology in our series is in keeping with the established association of the virus with NPC. Although EBV has been shown to be related to COX-2 expression, ${ }^{7}$ the similarity of the incidence of positive EBV serology in the COX-2 positive and negative groups in our series suggests that other factors are probably also necessary for COX-2 expression.

In some cancers, such as breast cancer, COX-2 expression is associated with adverse prognostic features, such as larger tumour size and lymph node metastases, in addition to reduced disease free survival. ${ }^{22}{ }^{23}$ Despite having a fairly large study size, we were not able to show an association between COX-2 expression and tumour TNM staging, which is an important prognostic factor in NPC.

Radiotherapy has been the mainstay of NPC treatment. More recently, chemotherapy has appeared to provide additional benefit to patients with advanced disease. ${ }^{24} \mathrm{COX}$ 2 inhibitors have been shown to potentiate the effects of radiotherapy and chemotherapy on tumour cells experimentally. ${ }^{25}{ }^{26}$ COX-2 inhibitors have been held to offer some promise in the chemoprevention and adjunctive treatment of squamous cell carcinomas of the head and neck in general. ${ }^{27}$ Thus, the role of COX-2 as a predictive factor for NPC, and of COX-2 inhibitors in the prevention and management of this cancer should be explored further. Other recent studies have shown that many NPCs overexpress the oncogene products, c-KIT and epidermal growth factor receptor, ${ }^{28}{ }^{29}$ both of which can be targeted by specific pharmacological agents. 


\section{Take home messages}

- Cyclooxygenase 2 (COX-2) was expressed in a high proportion of nasopharyngeal carcinomas (NPCs)

- COX-2 expression was more frequently seen as nasopharyngeal epithelium progressed from normal to dysplastic to carcinoma, although COX-2 positivity was not associated with a particular NPC subtype

- COX-2 probably contributes to the multistep process of NPC carcinogenesis

- Further investigations are needed into the use of COX2 inhibitors as adjuvant treatment in NPC, particularly in view of the fact that they potentiate the antitumour effects of radiotherapy, the primary treatment for NPC

Clinical trials to evaluate the potential for such agents, including COX-2 inhibitors, in the multimodality management of NPCs would be useful.

\section{ACKNOWLEDGEMENTS}

We thank Mr CK Ow and Ms SN Choo for the immunohistochemistry work and Mr TC Tan for the photography.

\section{Authors' affiliations}

K-B Tan, Department of Pathology, National University of Singapore, Lower Kent Ridge Road, Singapore 119074

T C Putti, Department of Pathology, National University of Singapore

This study was presented orally at Pathology Update 2004, annual meeting of the Royal College of Pathologists of Australasia, held in Sydney, Australia, 12-14 March 2004

\section{REFERENCES}

1 Gately S. The contributions of cyclooxygenase-2 to tumour angiogenesis. Cancer Metastasis Rev 2000;19:19-27.

2 Dannenberg AJ, Altorki NK, Boyle JO, et al. Cyclooxygenase-2: a pharmacological target for the prevention of cancer. Lancet Oncol 2001:2:544-51.

3 Steinbach G, Lynch PM, Phillips RK, et al. The effect of celecoxib, a cyclooxygenase-2 inhibitor, in familial adenomatous polyposis. N Engl J Med 2000;342:1946-52

4 Oshima M, Dinchuk JE, Kargman SL, et al. Suppression of intestinal polyposis in APCdelta716 knockout mice by inhibition of cyclooxygenase-2 (COX-2). Cell 1996;87:803-9.

5 Parkin DM, Whelan SL, Ferlay J, et al. Cancer incidence in five continents, Vol. VII. Scientific Publications no. 143. Lyon: IARC, 1997.

6 Bar-Sela G, Kuten A, Minkov I, et al. Prevalence and relevance of EBV latency in nasopharyngeal carcinoma in Israel. J Clin Pathol 2004;57:290-3.
7 Murono S, Inoue $\mathrm{H}$, Tanabe T, et al. Induction of cyclooxygenase- 2 by EpsteinBarr virus latent membrane protein 1 is involved in vascular endothelial growth factor production in nasopharyngeal carcinoma cells. Proc Natl Acad Sci U S A 2001;98:6905-10.

8 Greene FL, Page DL, Fleming ID, et al. Pharynoc (including base of the tongue, soft palate and uvula). In: AJCC cancer staging handbook. American Joint Committee on Cancer. New York: Springer, 2002:47-60.

9 Chan CW, Nicholls JM, Sham JST, et al. Nasopharyngeal carcinoma in situ in nasopharyngeal carcinoma. J Clin Pathol 1992;45:898-901.

10 Pathmanathan R, Prasad U, Sadler R, et al. Clonal proliferations of cells infected with Epstein-Barr virus in preinvasive lesions related to nasopharyngeal carcinoma. N Engl J Med 1995;333:693-8.

11 Lee JCK. Precancerous changes. In: van Hasselt CA, eds. Nasopharyngeal carcinoma. Hong Kong: Chinese University Press, 1991:37-45.

12 Pak MW, To KF, Lo YM, et al. Nasopharyngeal carcinoma in situ (NPCIS)pathologic and clinical perspectives. Head Neck 2002;24:989-95.

13 Sheu LF, Chen A, Meng CL, et al. Analysis of bcl-2 expression in normal, inflamed, dysplastic nasopharyngeal epithelia, and nasopharyngeal carcinoma: association with p53 expression. Hum Pathol 1997;28:556-62.

14 Tsujii M, DuBois RN. Alterations in cellular adhesion and apoptosis in epithelial cells overexpressing prostaglandin endoperoxide synthase 2. Cell 1995:83:493-501.

15 Soslow RA, Dannenberg AJ, Rush D, et al. COX-2 is expressed in human pulmonary, colonic, and mammary tumours. Cancer 2000;89:2637-45.

16 Shim V, Gauthier ML, Sudilovsky D, et al. Cyclooxygenase-2 expression is related to nuclear grade in ductal carcinoma in situ and is increased in its normal adjacent epithelium. Cancer Res 2003:63:2347-50.

17 Weiland LH. Nasopharyngeal carcinoma. In: Barnes L, eds. Surgical pathology of the head and neck. New York: Marcel Dekker, 1985:453-66.

18 Wenig BM, Pilch BZ. Tumors of the upper respiratory tract. In: Fletcher CDM, eds. Diagnostic histopathology of tumors, 2nd ed. London: Churchill Livingstone, 2000:87-169.

19 Tsujii M, Kawano S, Tsuji S, et al. Cyclooxygenase regulates angiogenesis induced by colon cancer cells. Cell 1998;93:705-16.

20 Masferrer JL, Leahy KM, Koki AT, et al. Antiangiogenic and antitumour activities of cyclooxygenase- 2 inhibitors. Cancer Res 2000;60:1306-11.

21 Tatsuguchi A, Matsui K, Shinji Y, et al. Cyclooxygenase-2 expression correlates with angiogenesis and apoptosis in gastric cancer tissue. Hum Pathol 2004:35:488-95.

22 Costa C, Soares R, Reis-Filho JS, et al. Cyclo-oxygenase 2 expression is associated with angiogenesis and lymph node metastasis in human breast cancer. J Clin Pathol 2002;55:429-34.

23 Ristimaki A, Sivula A, Lundin J, et al. Prognostic significance of elevated cyclooxygenase-2 expression in breast cancer. Cancer Res 2002;62:632-5.

24 Al-Sarraf M, LeBlanc M, Giri PG, et al. Chemotherapy versus radiotherapy in patients with advanced nasopharyngeal cancer: phase III randomized intergroup study 0099. J Clin Oncol 1998;16:1310-17.

25 Milas L, Kishi K, Hunter N, et al. Enhancement of tumour response to gammaradiation by an inhibitor of cyclooxygenase-2 enzyme. J Natl Cancer Inst 1999;91:1501-4

26 Moore RJ, Zweifel BS, Heuvelman DM, et al. Enhanced antitumour activity by co-administration of celecoxib and the chemotherapeutic agents cyclophosphamide and 5-FU. Proc Am Assoc Cancer Res 2000;41:409.

27 Lin DT, Subbaramaiah K, Shah JP, et al. Cyclooxygenase-2: a novel molecular target for the prevention and treatment of head and neck cancer. Head Neck 2002;24:792-9.

28 Bar-Sela G, Kuten A, Ben-Eliezer S, et al. Expression of HER2 and c-KIT in nasopharyngeal carcinoma: implications for a new therapeutic approach. Mod Pathol 2003;16:1035-40

29 Leong JL, Loh KS, Putti TC, et al. Epidermal growth factor receptor in undifferentiated carcinoma of the nasopharynx. Laryngoscope 2004;114:153-7. 